\title{
SPACE CHARGE AND BEAM STABILITY ISSUES OF THE FERMILAB PROTON DRIVER IN PHASE I
}

\author{
King-Yuen $\mathrm{Ng}^{1}$ and B. Zotter ${ }^{2}$ \\ ${ }^{1}$ Fermi National Accelerator Laboratory, ${ }^{*}$ P.O. Box 500, Batavia, IL 60510 \\ ${ }^{2}$ CERN, CH-1211, Geneva 23, Switzerland
}

(July 2001)

\begin{abstract}
Issues concerning beam stability of the proposed Fermilab Proton Driver are studied in its Phase I. Although the betatron tune shifts are dominated by space charge, these shifts are less than 0.25 and will therefore not drive the symmetric and antisymmetric modes of the beam envelope into instability. The longitudinal space charge force is large and inductive inserts may be needed to compensate for the distortion of the rf potential. Although the longitudinal impedance is space charge dominated, it will not drive any microwave instability, unless the real part of the impedance coming from the inductive inserts and wall resistivity of the beam tube are large enough. The design of the beam tube is therefore very important in order to limit the flow of eddy current and keep wall resistivity low. The transverse impedance is also space charge dominated. With the Proton Driver operated at an imaginary transition gamma, however, Landau damping will never be canceled and beam stability can be maintained with negative chromaticities.
\end{abstract}

* Operated by the Universities Research Association, Inc., under contract with the U.S. Department of Energy. 


\section{INTRODUCTION}

The Fermilab Proton Driver will be a rapid-cycling high intensity 16-GeV synchrotron that serves a number of purposes in the Fermilab high energy physics program. In Phase I, the Proton Driver is designed to provide $1 \mathrm{MV}$ of beam power, while in Phase II $4 \mathrm{MW}$. There are two stages in Phase I. Stage 1 provides a maximum beam kinetic energy of $12 \mathrm{GeV}$ with a $53 \mathrm{MHz}$ rf system to serve as an injector to the Main Injector, whereas Stage 2 increases the beam energy to $16 \mathrm{GeV}$ with a new $7.5 \mathrm{MHz}$ rf system to serve as a production source for muons destined for a neutrino factory. For a synchrotron of such high intensity, a thorough study of the beam stability issues is necessary. Protons are injected into the Proton Driver from the existing Fermilab linac at the kinetic energy of $400 \mathrm{MeV}$. Thus space charge problems can become important. In this article, we are going to study the space charge effects, betatron tune shifts, and coherent single-bunch and multi-bunch instabilities that may be relevant to the Proton Driver. The problems of a bunch rotation before extraction to produce short bunches as well as trapped electrons to produce e-p instability are also addressed. The discussion will be limited to Phase I. Some parameters of the Proton Driver are listed in Table I.

Table I: Some parameters of the Proton Driver in Stages 1 and 2 of Phase I operation.

\begin{tabular}{lrrl}
\hline & Stage 1 & Stage 2 & \\
\hline Circumference $C=2 \pi R$ & 711.3 & 711.3 & $\mathrm{~m}$ \\
Injection kinetic energy & 400 & 400 & $\mathrm{MeV}$ \\
Extraction kinetic energy & 12 & 16 & $\mathrm{GeV}$ \\
Repetition rate & 15 & 15 & $\mathrm{~Hz}$ \\
Total number of protons $N$ & $3 \times 10^{13}$ & $3 \times 10^{13}$ & \\
Number of bunches & 126 & 18 & \\
Protons per bunch $N_{b}$ & $5.238 \times 10^{12}$ & $1.67 \times 10^{12}$ & \\
Rf frequency & 53 & 7.5 & $\mathrm{MHz}$ \\
Longitudinal emittance & 0.1 & 0.4 & $\mathrm{eV}-\mathrm{s}$ \\
Extraction rms bunch length & 3 & 3 & $\mathrm{~ns}$ \\
Normalized $95 \%$ trans. emittance & $60 \times 10^{-6}$ & $60 \times 10^{-6}$ & $\pi \mathrm{m}$ \\
Betatron tunes $\nu_{x, y}$ & $12.428 / 11.380$ & $12.428 / 11.380$ & \\
Average betatron function $\left\langle\beta_{x, y}\right\rangle$ & $9.109 / 9.948$ & $9.109 / 9.948$ & $\mathrm{~m}$ \\
\hline
\end{tabular}




\section{SPACE CHARGE AND IMAGE EFFECTS}

\subsection{Incoherent Betatron Tune Shifts}

The betatron tunes $\nu_{z}, z=x$ or $y$ denoting horizontal or vertical, of transverse oscillations of charged particles are important parameters in the operation of a particle beam inside an accelerator ring. They are determined mainly by the applied focusing forces due to quadrupoles. With finite beam current the tunes are shifted, both by direct space charge and by "image" forces due to induced voltages in the surrounding structure impedances. These shifts, if large enough, may render the beam unstable. At relativistic beam energies, the space charge forces are strongly reduced by a factor $\gamma^{-2}=1-\beta^{2}$ due to partial compensation of electric and magnetic forces, where $v=\beta c$ is the axial velocity of the beam particles, with $c$ being the velocity of light. However, in the Proton Driver at $400 \mathrm{MeV}$ injection energy, $\gamma=1.426$ and the space charge term is then largely dominant.

The incoherent tune shift of a transversely uniform beam of elliptic cross section, with half width $a_{x}$ and half height $a_{y}$, consisting of $N$ protons with classical radius $r_{p}$, with bunching factor $B_{f}$, the ratio of average to peak current, circulating in a vacuum chamber of half height $h$, inside a magnet gap of half-height $g$ extending over a fraction $\kappa$ of the machine circumference, is given by $[1]$

$$
\Delta \nu_{z i n c}=-\frac{N r_{p}\left\langle\beta_{z}\right\rangle}{\pi \beta^{2} \gamma}\left[\frac{\gamma^{-2}-\chi_{e}}{B_{f} a_{z}\left(a_{x}+a_{y}\right)}+\left(\beta^{2}+\frac{\gamma^{-2}-\chi_{e}}{B_{f}}\right) \frac{\epsilon_{1 z}}{h^{2}}+\kappa \beta^{2} \frac{\epsilon_{2 z}}{g^{2}}\right],
$$

where $\left\langle\beta_{z}\right\rangle \approx R / \nu_{z}$ is the average value of the betatron function, $R$ the average radius of the ring, $\chi_{e}$ the fractional neutralization which reduces the electric but not the magnetic force, thus perturbing their compensation. The incoherent Laslett image coefficients $\epsilon_{1,2 z}$ describe the strength of image forces for a particular geometry. Some examples of the electric image coefficients $\epsilon_{1 z}$ are shown in the second and third columns of Table II. The magnetic image coefficients $\epsilon_{2 z}$ are not defined for a closed cross section. For a pair of horizontal parallel plates, however, they are $\epsilon_{1 y}=-\epsilon_{1 x}=\pi^{2} / 24$.

Except for large neutralizations at high energies (when $\chi_{e}>\gamma^{-2}$ ), the space charge tune shift, the first term in Eq. (2.1), is always negative. Since the vertical incoherent Laslett coefficients are positive, these image terms add to the space charge tune shift, while they reduce it in the horizontal direction.

The beam is chopped when injected into a rf bucket of the Proton Driver from the 
Table II: Laslett image coefficients at the center of various cross sections. The aspect ratio is 119:63 for the elliptic and rectangular cross sections.

\begin{tabular}{|c||c|c|c|c|c|c|}
\hline Geometry & $\epsilon_{1 y}$ & $\epsilon_{1 x}$ & $\xi_{1 y}$ & $\xi_{1 x}$ & $\xi_{1 y}-\epsilon_{1 y}$ & $\xi_{1 x}-\epsilon_{1 x}$ \\
\hline \hline parallel plates & 0.2056 & -0.2056 & 0.6169 & 0.0000 & 0.4112 & 0.2056 \\
rectangular & 0.1926 & -0.1926 & 0.6039 & 0.0261 & 0.4113 & 0.2187 \\
elliptic & 0.1669 & -0.1669 & 0.5956 & 0.0948 & 0.4287 & 0.2617 \\
circular & 0.0000 & -0.0000 & 0.5000 & 0.5000 & 0.5000 & 0.5000 \\
\hline
\end{tabular}

linac. The bunching factor is $B_{f}=0.9$. The bunch length and transverse dimensions are reduced during the acceleration cycle at the repetition rate of $15 \mathrm{~Hz}$. These were obtained by computer simulation and the bunching factor together with the kinetic energy are plotted in Fig. 1 for the acceleration cycle [2]. The evolution of tune shifts are then calculated from Eq. (2.1). The results for $\chi_{e}=0$ are shown in Fig. 1 and Table III. For

Table III: Space charge, image, incoherent, and coherent tune shifts.

\begin{tabular}{|r||c|c|c|c||c|c|c|c|}
\hline$t(\mathrm{~ms})$ & $\Delta \nu_{y}^{s c}$ & $\Delta \nu_{\text {yinc }}^{\text {im }}$ & $\Delta \nu_{\text {yinc }}$ & $\Delta \nu_{y c o h}$ & $\Delta \nu_{x}^{\text {sc }}$ & $\Delta \nu_{\text {xinc }}^{\text {im }}$ & $\Delta \nu_{\text {xinc }}$ & $\Delta \nu_{x c o h}$ \\
\hline \hline 0 & -0.0956 & -0.0140 & -0.1096 & -0.0260 & -0.0915 & 0.0139 & -0.0786 & 0.0062 \\
1 & -0.1749 & -0.0176 & -0.1924 & -0.0388 & -0.1673 & 0.0176 & -0.1512 & 0.0042 \\
2 & -0.1699 & -0.0165 & -0.1863 & -0.0357 & -0.1625 & 0.0164 & -0.1474 & 0.0043 \\
5 & -0.1200 & -0.0110 & -0.1310 & -0.0207 & -0.1148 & 0.0109 & -0.1047 & 0.0047 \\
10 & -0.0481 & -0.0049 & -0.0530 & -0.0069 & -0.0460 & 0.0048 & -0.0415 & 0.0034 \\
20 & -0.0155 & -0.0020 & -0.0174 & -0.0023 & -0.0148 & 0.0019 & -0.0130 & 0.0017 \\
30 & -0.0068 & -0.0012 & -0.0080 & -0.0012 & -0.0065 & 0.0011 & -0.0055 & 0.0010 \\
38 & -0.0055 & -0.0010 & -0.0065 & -0.0010 & -0.0052 & 0.0010 & -0.0043 & 0.0009 \\
\hline
\end{tabular}

small neutralizations the incoherent tune shifts are actually reduced. In the computation, the vacuum chamber has been assumed to be made of stainless steel having an elliptical cross section with horizontal/vertical radii $w=119 \mathrm{~mm}$ and $h=63 \mathrm{~mm}$. The bare horizontal/vertical tunes are $\nu_{0 x, y}=12.428 / 11.380$ so that the average betatron functions are $\left\langle\beta_{x, y}\right\rangle=9.109 / 9.948 \mathrm{~m}$. The average dispersion is $\langle D\rangle=1.247 \mathrm{~m}$. The beam has normalized $95 \%$ emittance $\epsilon_{x, y}=60 \times 10^{-6} \pi \mathrm{m}$. Thus, at injection the beam has elliptical cross section with horizontal/vertical radii $a_{x, y}=23.2 / 24.2 \mathrm{~mm}$, where the full energy spread is $7 \times 10^{-4}$ throughout the cycle. The rf frequency for Stage 1 is $53 \mathrm{MHz}$. The magnet half gap is 


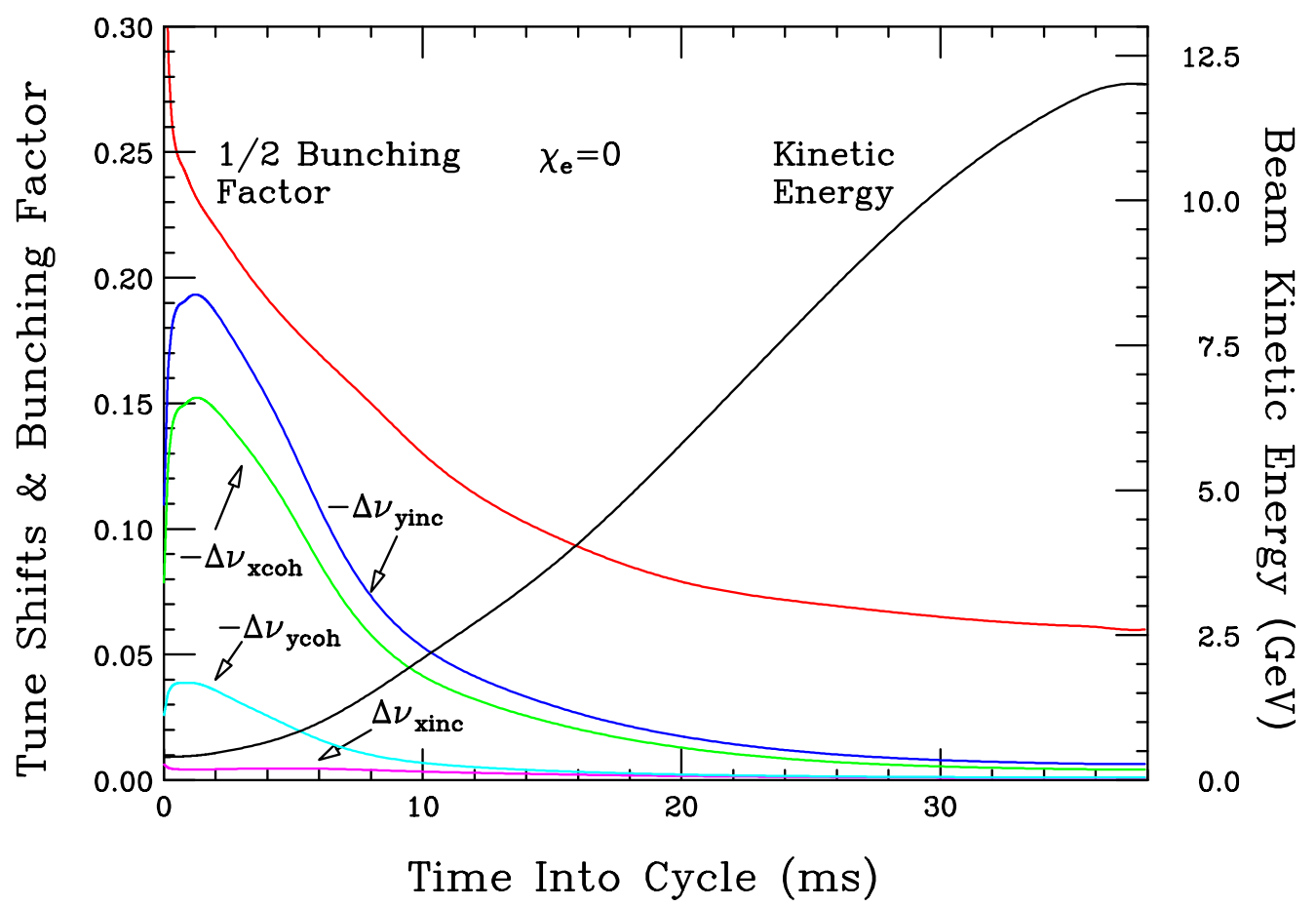

Figure 1: (color) Bunching factor, energy and tune shifts during one acceleration cycle.

$g=64 \mathrm{~mm}$ covering $\kappa=0.5$ of the ring. For the magnet pole faces, the parallel-plate model was used in the computation of the magnetic images coefficients.

We see that the largest incoherent tune shifts occur 1-2 ms after injection, when the beam energy is still low, but the bunch length had decreased strongly as the protons were captured in the rf field.

It is usually considered prudent to keep all tunes away from low-order resonances, in particular from integer multiples of the revolution frequency. Therefore conservative tune shift limits of 0.25 are often assumed, and it is satisfying that the estimates given above do not exceed this limit. However, if the transverse distribution of particles is not uniform but peaked, the space charge tune shift can be much larger. For example, for those particles in the center of the beam, the betatron tune is shifted three times larger with bi-Gaussian distribution than with uniform distribution. Computer simulation had been performed [3] and the results show that the incoherent tune shifts were slightly above 0.25 in the vertical, and just below it in the horizontal direction. However, since the dominant space charge force is generated inside a particle beam itself, it actually does not deflect the beam center 
and thus cannot drive the whole beam into dipole resonances [4]. This can be seen from the equation for betatron oscillations of a particle with offset $y$ from the center of the vacuum chamber, in the presence of space charge forces $F_{s c}$ and other forces $F_{i m}$ coming from images and vacuum chamber discontinuity:

$$
\frac{d^{2} y}{d t^{2}}+\left(\nu_{y} \omega_{0}\right)^{2} y=F_{i m} y+F_{s c}(y-\bar{y})
$$

where $\bar{y}$ is the offset of the beam center. Averaging over all particles yields

$$
\frac{d^{2} \bar{y}}{d t^{2}}+\left(\nu_{y} \omega_{0}\right)^{2} \bar{y}=F_{i m} \bar{y}
$$

i.e., the driving term for the space-charge effect vanishes, while the image term causes a shift of the coherent tune to $\nu_{c o h}^{2}=\nu_{y 0}^{2}-F_{i m} / \omega_{0}^{2}$.

\subsection{Coherent Betatron Tune Shifts}

The coherent tune shifts are given by the same equations as the incoherent ones, but without the space charge term and with the Laslett incoherent image coefficients $\epsilon_{1,2 z}$ replaced by the coherent counterparts $\xi_{1,2 z}$. However, for the usual case of a chamber wall thick enough and the betatron tunes near half integers, the ac magnetic field arising from betatron oscillation does not penetrate the vacuum chamber and we have the coherent tune shift [5],

$$
\Delta \nu_{z}=-\frac{N r_{p}\left\langle\beta_{z}\right\rangle}{\pi \beta^{2} \gamma}\left[\beta^{2} \frac{\epsilon_{1 z}}{h^{2}}+\frac{\gamma^{-2}-\chi_{e}}{B_{f}} \frac{\xi_{1 z}}{h^{2}}+\kappa \beta^{2} \frac{\epsilon_{2 z}}{g^{2}}\right] .
$$

Notice that the coherent magnetic image coefficient $\xi_{2 z}$ is made up of the dc part $\epsilon_{2 z}$ and the ac part which is the rest. Since only the dc part penetrates the vacuum chamber to land on the pole faces, we therefore have $\epsilon_{2 z}$ in the magnet pole term. Since only the ac magnetic field from betatron oscillation is stopped at the vacuum chamber, the dc part is being subtracted, which gives the first term inside the squared brackets. Some values of the coherent image coefficients are listed in Table II.

The direct space charge force does not affect dipole oscillations, but it does change the external focusing forces. In one dimension, the evolution of the beam size $a_{z}(z=x$ or $y)$ is described by the envelope equation [6]

$$
\frac{d^{2} a_{z}}{d s^{2}}+K_{z}(s) a_{z}-\frac{\epsilon_{z}}{a_{z}^{3}}=\frac{4 \lambda r_{p}}{\beta^{2} \gamma^{3}\left(a_{x}+a_{y}\right)},
$$


where $K_{z}(s)$ is the external focusing strength, $\epsilon_{z}$ the unnormalized transverse emittance, and $\lambda$ the number of particles per unit length.

The space charge force leads to a modulation of the beam envelope which reduces the tune shift of the lowest quadrupole mode to $\frac{3}{4} \nu_{s c}$, where $\nu_{s c}$ is the tune shift of the linearspace-charge-equivalent beam. For a uniformly distributed beam, $\nu_{s c}$ is just the self-field tune shift which is the same for all particles, while for a bi-Gaussian distribution, it is one half of the maximum self-field tune shift for those particles at the beam center. For the 2dimensional case, there are 2 modes of quadrupole oscillations: the antisymmetric mode has its tune shift also reduced to $\frac{3}{4} \nu_{s c}$, and the symmetric mode even to $\frac{1}{2} \nu_{s c}$. Hence these modes are not excited when only the incoherent tune crosses (half-)integer resonances. Nevertheless, one has to allow for a variation of tunes during acceleration due to incomplete tracking of quadrupole and dipole strengths, and therefore one needs a certain safety margin to these resonances. In Phase I of the Proton Driver, tune shifts do not exceed 0.25, which can be safely accommodated with a properly chosen working point.

Higher order oscillations - sextupole, octupole etc.- have larger space charge tune shifts, but have not been observed in simulation nor in actual machines. They are suppressed by Landau damping due to the non-linearity of space charge forces.

\subsection{Longitudinal space charge effects}

The coherent synchrotron frequency of a bunch is nearly constant with current since the coherent and incoherent longitudinal tune shifts cancel, $\Delta \nu_{s, c o h}=-\Delta \nu_{s, i n c}$. At low energies the incoherent frequency shift of Gaussian beam can be written [6]

$$
\Delta \nu_{s, i n c}=-\frac{3 N r_{p} \eta R^{2}}{2 \beta^{2} \gamma^{3} \nu_{s 0}} \frac{g_{f}}{L_{b}^{3}},
$$

where $L_{b}$ is the full bunch length, and $g_{f}$ is commonly known as the $g$-factor. For a circular beam of radius $a$ in a concentric chamber of radius $b$ it is $g_{f}=2 \ln (b / a)+1 / 2$, for a rectangular chamber $b$ should be replaced by $4 h / \pi$. This expression contains both the contribution of space charge in the term $\ln a$ and that of the wall in the term $\ln b$.

The voltage induced by the bunch current creates a local potential-well distortion which slides up and down the applied rf voltage when the synchronous phase angle changes with bunch current. The (coherent) synchrotron frequency of the bunch, which depends on the 
derivative of the rf voltage, therefore remains constant as long as the applied voltage is sufficiently linear. However, the (incoherent) synchrotron frequency of individual particles, given by the local derivative inside the potential well, not only depends on beam current but also varies between the center and the edge of a bunch. When the (square of the) synchrotron frequency in the center of a bunch is shifted below zero, an instability may occur, hence excessive potential well deformations should be avoided.

The mainly inductive wall impedance reduces the tune shift of the "capacitive" space charge, but is insufficient to compensate it in particular at injection energy. Thus it is useful to add more inductance, which can be done e.g. with inductive inserts, containing ferrite or Finemet cores [9]. Even at higher energies the space charge tune shift may be large if the bunch length is sufficiently small, e.g. due to reduced transition energy or rotation in phase space (see below). Again inductive inserts can be helpful. However, one has to take care that the total resistive part of the impedance is not increased excessively by them, as this determines the growth-rate of instabilities (see next section).

\subsection{Effects of space charge on bunch rotation}

In Phase I Stage 2 and Phase II, it is desirable to have short proton bunches (1-2 ns) impinging on the target for efficient production of muons and hence neutrinos. Therefore it was proposed to rotate the bunches in phase space just prior to ejection, converting their small energy spread into a short bunch length.

The minimum bunch length thus achievable is restricted by distortions of the bunch during phase space rotation. The speed of rotation of individual particles is given by their synchrotron tunes:

$$
\nu_{s}=\sqrt{\frac{-\eta h V_{\mathrm{rf}} \cos \phi_{s}}{2 \pi E / e}},
$$

where $V_{\mathrm{rf}}$ is the applied rf voltage at frequency $f_{\mathrm{rf}}$ with harmonic number $h=f_{\mathrm{rf}} / f_{0}$, $f_{0}=\omega_{0} /(2 \pi)$ being the revolution frequency, and synchronous phase $\phi_{s}$. The slip factor

$\eta=\gamma_{T}^{-2}-\gamma^{-2}$ expresses the distance to transition energy, and is negative below transition (for which $\cos \phi_{s}$ is therefore chosen positive). $\eta$ is only a weak function of energy when $\gamma$ is not too close to $\gamma_{T}$, and then the variation of synchrotron frequency becomes only important for beams with large momentum spreads. 
In the Proton Driver, the full momentum spread is usually quite small $\left(7.10^{-4}\right)$. However, it has been proposed to reduce transition energy prior to ejection to shorten the bunches and require only little rotation in phase space. Strong distortions may result during bunch rotation due to the increased synchrotron frequency spread [7].

\section{Longitudinal effects}

For an intense proton bunch, the longitudinal space charge force will counteract the focusing rf force, thus reducing the synchrotron tune and slowing down the rotation rate. This cancellation becomes larger and larger as the bunch becomes shorter and shorter during the rotation. Sometimes, this space charge force will even be larger than the rf focusing force, making particles embark on an unstable hyperbolic trajectory. However, this may not be important, because we are interested in only about $\frac{1}{4}$ of a synchrotron period. The space charge modification of the rf potential occurs only near the core of the bunch where the particle intensity and therefore space charge is most intense. Ironically, this longitudinal space charge force is actually beneficial to the bunch rotation. This is because the slowing down of the rotation near the core provides time for the particles near the separatrices to catch up. As a result, the fraction of particles in the tails of the rotated compressed bunch will be much less. Of course, when the space charge is too large, bunch lengthening dominates because of the hyperbolic trajectories of the core particles and bunch compression becomes impossible. Figure 2 shows the simulation result of the minimum bunch length acquired through such a rotation as a function of space charge impedance for a typical bunch in the Cooler Ring at IUCF. From Fig. 2, it appears that in order to have a final compressed bunch length $\sigma_{\tau} \lesssim 3.85 \mathrm{~ns}$ (the minimum bunch length when space charge is absent), the space charge impedance per harmonic must be limited to $|Z / n|_{s c} \lesssim 15000 \Omega$. In other words, the ratio of the space charge force to the rf force must be less than the critical value of [10]

$$
\left.\frac{\text { Sp-ch force }}{\text { Rf force }}\right|_{\text {critical }}=\frac{e N_{b}|Z / n|_{s c}}{\sqrt{2 \pi} h \omega_{0}^{2} \sigma_{\tau}^{3} V_{\mathrm{rf}}} \sim 22.0,
$$

where $N_{b}$ is the number of particles in the bunch, $(Z / n)_{s c}$ the longitudinal space charge impedance per harmonic, $V_{\mathrm{rf}}$ the maximum $\mathrm{rf}$ voltage at $\mathrm{rf}$ harmonic $h, \omega_{0} /(2 \pi)$ the revolution frequency, and $\sigma_{\tau}$ the desired rms bunch length after compression. It is important to point out that the actual space charge impedance of the IUCF Cooler is only $|Z / n|_{s c} \approx 1500 \Omega$. What we are saying is that, while a rms bunch length $\sigma_{\tau}=3.85$ ns can be obtained in the absence of space charge, a space charge impedance as large as $|Z / n|_{s c} \approx 15000 \Omega$ will not lead to a longer compressed rms bunch length although the rf potential will be severely 


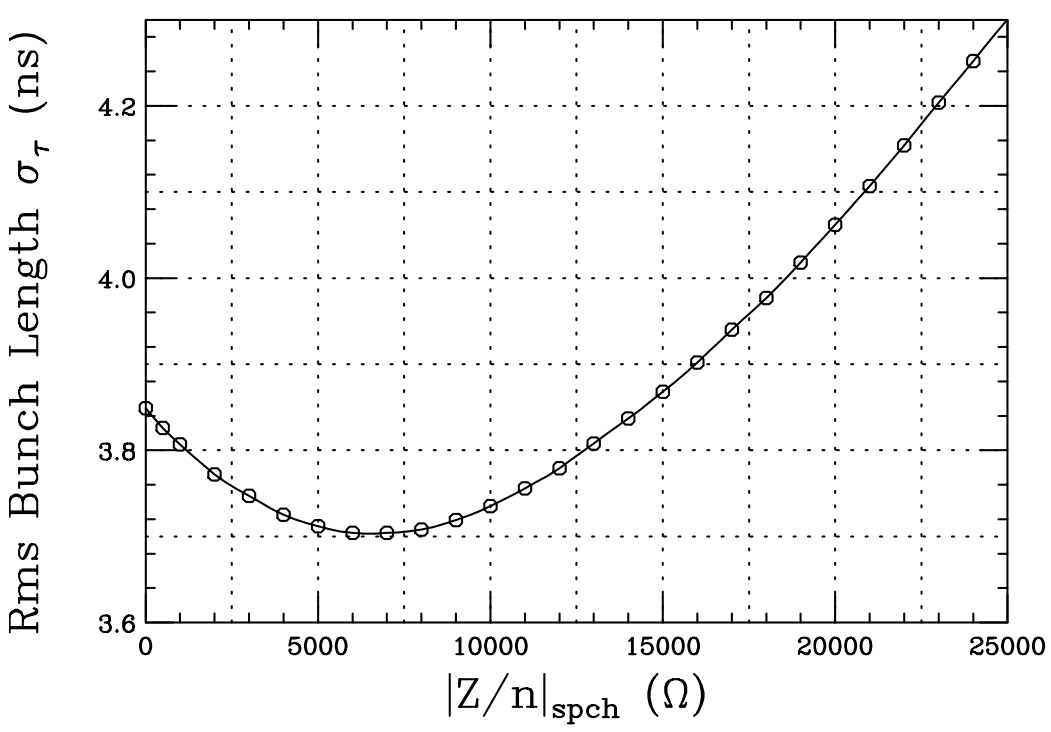

Figure 2: Plot showing shortest rms bunch length $\sigma_{\tau}$ obtained through rotation as a function of the space charge impedance for a typical bunch in the Cooler Ring at IUCF.

distorted.

The IUCF experiment is compared with the Fermilab Proton Driver in Table IV. It is evident that for Phase I Stage 2 of the Fermilab Proton Driver, the space charge-to-rf ratio is very much less than the critical value of 22 stated in Eq. (2.8), implying that the longitudinal space charge constitute negligible influence on the bunch compression. Thus, we expect the bunch compression will not be affected longitudinally by the space charge force. For Phase II operation, the space charge-to-rf ratio for Phase II operation of the Fermilab Proton Driver is roughly at the critical value. Thus, we expect a compression to $\sigma_{\tau}=1 \mathrm{~ns}$ is still possible.

A shortcoming of the bunch rotation method is the possible development of microwave instability when the rf voltage is reduced adiabatically to a small value so that the bunch will fill the whole bucket prior to the rotation. This can be avoided if the synchronousphase-shift method of compression is used instead. The synchronous phase is first shifted from the center of the bucket to an unstable fixed point. The bunch is allowed to spread out along one set of separatrices. Later the synchronous phase is shifted back to the center of the bucket. The bunch is allowed to rotate in the longitudinal phase space for about $\frac{3}{8}$ of a synchrotron period and the shortest bunch results. This method gives a theoretical linear compression ratio of $\sqrt{2} /\left(\sqrt{3} \sigma_{\phi}\right)$, where $\sigma_{\phi}$ is the initial rms bunch length measured in $\mathrm{rf}$ phase [11]. Of course, final rotation will introduce nonlinearity and tails for the compressed bunch. However, this can be alleviated by extracting the bunch immediately at the end of 
Table IV: Comparison of the space charge-to-rf ratio in bunch compression through rotation for the IUCF Cooler Ring, Phase I Stage 2 and Phase II of the Fermilab Proton Driver.

\begin{tabular}{|l||r|r|r|}
\hline & IUCF & \multicolumn{2}{|c|}{ Fermilab Proton Driver } \\
\cline { 3 - 4 } & Critical & Phase I & Phase II \\
\hline \hline Ring circumference $(\mathrm{m})$ & 86.83 & 711.32 & 711.32 \\
Extraction kinetic energy $(\mathrm{GeV})$ & 0.203 & 16 & 16 \\
Number per bunch $N_{b}$ & $1.010^{9}$ & $1.710^{12}$ & $2.510^{13}$ \\
Revolution frequency $(\mathrm{MHz})$ & 1.97 & 0.40932 & 0.40932 \\
$|Z / n|_{s c}($ Ohms $)$ & 15000 & 2.639 & 2.639 \\
Maximum Rf voltage $V_{\text {rf }}(\mathrm{kV})$ & 1.0 & 1400 & 1400 \\
RF harmonic $h$ & 5 & 18 & 18 \\
Extraction $\sigma_{\tau}(\mathrm{ns})$ & 3.85 & 3 & 1 \\
\hline \hline Sp-ch-to-rf ratio & 22.0 & 0.060 & 23.9 \\
\hline
\end{tabular}

the drift along the separatrices. The bunch is then sheared back to an upright position in the beam line via a lengthy optic system with local momentum compaction, or the $R_{56}$ element of the transfer matrix.

\section{Transverse effects}

At the end of the bunch rotation in the longitudinal phase space, the bunch will be compressed to its minimum length of, for example, $\sigma_{\tau}=1 \mathrm{~ns}$ with half momentum spread $\delta= \pm 0.0482$, where a bunch area of $2 \mathrm{eV}$-s has been assumed. (Actually, the momentum aperture of the Proton Driver is less than \pm 0.025 . Thus to compress a bunch to $1 \mathrm{~ns}$, the bunch area must be tailored to less than $1 \mathrm{eV}$-s to begin with.) Although the extraction energy is high, the self-field space charge tune shift $\Delta \nu_{s c}$ given by the first term of Eq. (2.1) can still be appreciable. It is possible that the reduction in betatron tune can modify the effective transition gamma $\gamma_{T}$ to such an extent that particles find themselves near transition. Higher order momentum compaction will be needed because of the large momentum spread. This may result in ruining the whole bunch rotation procedure as a result of nonlinearity. 
Notice that the self-field space charge tune shift in Eq. (2.1) is inversely proportional to $\gamma^{3} \beta^{2}$. Thus, the tune shift is momentum dependent and can be written as

$$
\Delta \nu_{z} \approx \Delta \nu_{s c}\left(1-3 \delta+12 \delta^{2}\right)
$$

where $\Delta \nu_{s c}$ is evaluated at the nominal momentum. It is evident that the last two terms represent the first two lowest orders of chromaticity generated by the transverse space charge force. In a Hamiltonian formalism, $\nu_{z}=\partial H / \partial J_{z}$, where $J_{z}(z=x$ or $y)$ is the transverse betatron action, which is related to the transverse offset $z$ from the off-momentum closed orbit by $z=\sqrt{2 \beta_{z} J_{z}}$, and the unnormalized emittance $\epsilon$ by $\epsilon=2 J_{z}$. For the simple case of a Kapchinskij-Vladimirskij (KV) beam [12] where the transverse distribution is uniform, ${ }^{\dagger}$ $\Delta \nu_{s c}$ is $J_{z}$ independent. Thus, the contribution of the self-field space charge tune shift to the Hamiltonian is [10]

$$
\Delta H=\Delta \nu_{s c}\left(J_{x}+J_{y}\right)\left(1-3 \delta+12 \delta^{2}\right)-\frac{1}{2} \Delta \alpha_{s c} R \delta^{2} .
$$

The first term gives the tune shifts and chromaticities provided by space charge. The last term is called Umstätter effect ${ }^{\ddagger}$. It is the modification of the momentum compaction factor by space-charge tune shifts through the lattice. Although $\Delta \alpha_{s c}$ can be momentum dependent, it must be amplitude independent. If not, the space-charge tune shifts will be altered. For a FODO lattice, the change in transition gamma is roughly equal to the horizontal spacecharge tune shift (exact for a uniform focusing lattice). For a flexible momentum compaction lattice, this term can be very much smaller. The additional chromaticities are

$$
\Delta \xi_{x}=-3 \Delta \nu_{s c}(1-8 \delta), \quad \Delta \xi_{y}=-3 \Delta \nu_{s c}(1-8 \delta)
$$

The additional changes in path length and $\gamma_{T}$ are

$$
\begin{gathered}
\frac{\Delta \ell_{0}}{C}=\frac{1}{R}\left\langle\frac{d \Delta \ell}{d \theta}\right\rangle=-\frac{1}{R} \frac{\partial \Delta H}{\partial \delta}=\Delta \nu_{s c} \frac{J_{x} J_{y}}{R}(3-24 \delta)-\left[\frac{2 \Delta \nu_{s c}}{\gamma_{T}^{3}} \delta \cdots\right] \\
\Delta \gamma_{T} \approx 12 \gamma_{T}^{3} \Delta \nu_{s c} \frac{J_{x}+J_{y}}{R}+\Delta \nu_{s c}
\end{gathered}
$$

where $\theta$ is the independent 'time' variable which advances by $2 \pi$ for every revolutionary turn.

\footnotetext{
${ }^{\dagger}$ Even with other more realistic distributions, the result of the following discussions will not be much altered (see Ref. [10]).

$\ddagger$ This term has been mistakenly left out in Chapter 4 (p. 4-8) of The Proton Driver Design Study, Ed. W. Chou and C. Ankenbrandt, Fermilab Report TM-2136, 2000.
} 
In Phase II of the proton driver, the number per bunch is $N_{b}=2.5 \times 10^{13}$ and $\mathrm{rf}$ harmonic $h=18$. For the $\sigma_{\tau}=1$ ns compressed bunch, the bucket bunching factor is $B_{f} \approx$ $\sqrt{2 \pi} h f_{0} \sigma_{\tau}=0.01899$. With normalized $95 \%$ emittance $\epsilon_{\mathrm{N} 95}=60 \times 10^{-6} \pi \mathrm{m}$ and an average betatron function of $\left\langle\beta_{x}\right\rangle=10 \mathrm{~m}$, the self-field space-charge tune shift is $\Delta \nu_{s c}=-0.297$ at extraction. The maximum actions for betatron motion are $J_{x}=J_{y}=1.67 \times 10^{-6} \mathrm{~m}$. With the $2 \%$ momentum aperture in the vacuum chamber and the nominal transition gamma of $\gamma_{T}=j 27.71$, the maximum contributions to the additional fractional path difference are $2.62 \times 10^{-8}$ for the first term of Eq. (2.12) and $5.57 \times 10^{-7}$ for the second. The maximum rf voltage used during the bunch rotation is $V_{\mathrm{rf}}=1.4 \mathrm{MV}$, giving a synchrotron tune of

$\nu_{s}=1.02 \times 10^{-3}$. Thus during the $\frac{1}{4}$-synchrotron-period bunch rotation, the total cumulative maximum additional path difference due to space-charge tune shift is $0.32 \times 10^{-6}$ for the first term and $4.56 \times 10^{-5}$ for the second term. On the other hand, the ratio of the rms bunch length at extraction to the ring circumference is $\sigma_{\tau} / T_{0}=42.11 \times 10^{-5}$, which is much larger, implying that the effect of space-charge tune shift on bunch compression through rotation is very minimal.

It is important to point out that by having the $J_{z} \delta^{2}$ term in the additional Hamiltonian [Eq. (2.10)], we must include the same term into the original space-charge free Hamiltonian. This is the next order chromaticity, which will contribute a down-shift to $\gamma_{T}$ just like the first term Eq. (2.13) with $\Delta \nu_{s c}$ replaced by $\frac{1}{24}\left(\xi_{x 1} J_{x}+\xi_{y 1} J_{y}\right)$, where $\xi_{z}=\xi_{z 0}+\xi_{z 1} \delta+\cdots$. For a linear machine, $\xi_{z 1}=-2 \xi_{z 0}$. Thus, this order of chromaticity can lead to a much larger spread in $\gamma_{T}$ than the contribution from the space charge, and may require correction to ensure the bunch rotation.

\section{COHERENT SINGLE BUNCH INSTABILITIES}

\subsection{Broad-band impedance estimates}

The largest impedances in the Proton Driver, in particular at low energies, are due to space charge. They can be obtained from the last section [22]

$$
\frac{Z_{\|}^{s c}}{n}=-j \frac{Z_{0}}{2 \beta \gamma^{2}} g_{f}, \quad Z_{\perp z}^{s c}=-j \frac{2 R Z_{0}}{\beta^{2} \gamma^{2}}\left[\frac{1}{a_{z}\left(a_{x}+a_{y}\right)}-\frac{\xi_{1 z}-\epsilon_{1 z}}{b^{2}}\right] .
$$

The $g$-factor $g_{f}$ was already given after Eq. (2.6). For an elliptical chamber with 63 to 119 ratio, the Laslett coefficients yield $\xi_{1}-\epsilon_{1}=0.4287 / 0.2617$ vertically/horizontally, (for 
a rectangular one $0.4113 / 0.2187$. For a beam of radius $a=25 \mathrm{~mm}$, the longitudinal impedance at injection energy is then about $-387 j \Omega$, while the transverse one reaches nearly $-70 j /-63 j \mathrm{M} \Omega / \mathrm{m}$ horizontally/vertically.

The finite conductivity of the vacuum chamber wall creates the resistive wall impedance which contributes the largest real part to the impedance. It increases when the wall is made of high-resistance material such as Inconel or Ti alloys in order to reduce eddy currents.

For a material with conductivity $\sigma_{c}$, permeability $\mu=\mu_{r} \mu_{0}$, the skin depth at frequency $\omega$ is $\delta_{b}=\sqrt{2 /\left(\omega \mu \sigma_{c}\right)}$. For a wall thickness larger than the skin depth, the longitudinal resistive wall impedance, divided by mode number $n=\omega / \omega_{0}$, of a circular cylindrical wall at radius $b$ becomes

$$
\frac{Z_{\|}^{r w}}{n}=(1+j) \beta \mu_{r} Z_{0} \frac{\delta_{b}}{2 b},
$$

assuming that the wall is thick compared to the skin depth. The transverse impedance is found simply by multiplication with $2 R /\left(\beta b^{2}\right)$. The skin depths for various materials, evaluated at $300 \mathrm{kHz}$, the revolution frequency $f_{0}$ at injection energy, are shown in Table $\mathrm{V}$. The lowest betatron frequencies are smaller, $f_{0}$ multiplied by the non-integer part of the tune $q$ or $(1-q)$ if $q>\frac{1}{2}$. For simplicity, we give the skin depths and all impedances for the revolution frequency at injection energy. The transverse impedances have to be increased by the factor $q^{-1 / 2}$ or $(1-q)^{-1 / 2}$ once the exact tune is known. If the walls were made of

Table V: Resistive wall and space charge impedances at injection energy.

\begin{tabular}{|c||c|c|r|c||c|c|c|}
\hline Resistive wall & $\rho_{c}$ & $\sigma_{c}$ & \multicolumn{1}{c|}{$\mu_{r}$} & $\delta_{b}$ & $Z_{\|} / n$ & $Z_{\perp x}$ & $Z_{\perp y}$ \\
\hline Material & {$[\mu \Omega \mathrm{cm}]$} & {$[\mathrm{MS} / \mathrm{m}]$} & & {$[\mathrm{mm}]$} & {$[\Omega]$} & {$[\mathrm{k} \Omega / \mathrm{m}]$} & {$[\mathrm{k} \Omega / \mathrm{m}]$} \\
\hline \hline Silver & 1.59 & 62.9 & 1.0 & 0.116 & 0.247 & 5.53 & 19.7 \\
Copper OF & 1.71 & 58.5 & 1.0 & 0.120 & 0.256 & 5.74 & 20.5 \\
Aluminum & 2.91 & 34.4 & 1.0 & 0.157 & 0.334 & 7.49 & 26.7 \\
SS Steel & 57.1 & 1.75 & 1.001 & 0.694 & 1.480 & 33.2 & 118.4 \\
Si-Steel 1\% & 23 & 4.35 & 9000 & 0.005 & 89.05 & 1997 & 7124 \\
Inconel & 129 & 0.775 & 1.002 & 1.042 & 2.225 & 49.9 & 178.0 \\
Ti alloy & 148 & 0.676 & 1.0 & 1.117 & 2.381 & 53.4 & 190.5 \\
\hline \hline Space charge & & & & & $-j 384$ & $-j 69520$ & $-j 62870$ \\
\hline
\end{tabular}

good conductors, such as copper or aluminum, eddy current losses would be excessively high 
unless the metal was divided into narrow strips or wires, similar to the wire-cage used in ISIS [16]. The eddy current power loss per unit length in a metal strip of width $w$, height $h$ and conductivity $\sigma_{c}$, at right angle to a changing magnetic field with time derivative $\dot{B}$, is approximately given by $P / L=\sigma_{c} h w^{3} \dot{B}^{2} / 12$. Since it is proportional to the third power of the width, it can be reduced from over $8 \mathrm{~kW} / \mathrm{m}$ for a $1.3 \mathrm{~mm}$ thick, $22 \mathrm{~cm}$ wide, elliptic Inconel chamber to a few $\mathrm{W} / \mathrm{m}$ by replacing it with $2 \times 50$ copper strips of $4 \mathrm{~mm}$ width and 50-100 $\mu \mathrm{m}$ thickness. For a metal thickness $t$ small compared to the skin depth $\delta_{b}$, the impedance given by Eq. (3.2) has to be multiplied by a factor $\delta_{b} / t>1$. For the present case, this yields an impedance increase by about 3 for strips of $50 \mu \mathrm{m}$ thickness, still tolerable due to the higher conductivity of copper.

For vacuum chambers made of ceramics, thin metal stripes of high conductivity can be deposited on the inside to shield the high impedance of the magnetic pole pieces behind them. However, such chambers need a thickness of 5-6 mm to withstand the pressure of air and thus would require larger magnet gaps for the full aperture. Composite chambers can be made slightly thinner, with a thickness of $2-3 \mathrm{~mm}$, and would thus be preferable if their vacuum properties are found adequate.

Other sources of broad-band impedance are the rf cavities loaded with Ferrite (or Finemet). Kicker tanks may create both broad-band and narrow-band impedances. Finally, bellows and other small cross-section variations of the vacuum chamber become important when they are present in large numbers, but have essentially mainly inductive impedance at low frequencies.

\subsection{Shielding of electromagnetic fields by liners and cages}

Although the skin depth is smaller for larger permeability, the additional factor $\mu_{r}$ in Eq. (3.2) makes the impedance large for magnetic material such as used for iron pole pieces. In particular if the pole pieces form part of the vacuum chamber as in the Fermilab booster, they should be shielded for the driver where higher beam currents are desired. For this purpose, a screen or liner has been proposed, similar to the one being built as radiation shield for the LHC [15]. But to minimize eddy current losses, the screen for the driver should be made as thin as possible.

Therefore it is important to estimate the minimum thickness required to effectively shield the beam from the outer region. Assuming rotational symmetry, a screen of thickness 
$t$ at radius $b$, with skin depth $\delta_{b} \gg t$, and an outer wall at radius $d$, with skin depth $\delta_{d}$, the shielding condition in the longitudinal direction can be written [21]

$$
\frac{t}{\delta_{b}} \gg \frac{\beta^{2} \gamma^{2}}{2 \ln (d / b)} \frac{\delta_{b}}{b} \approx \frac{\beta^{2} \gamma^{2}}{2} \frac{\delta_{b}}{d-b}
$$

where the second relation holds when $d-b \ll b$. At low energies, when $\beta \gamma$ is small, the required screen thickness $t$ can thus be smaller than the skin depth by the factor $\delta_{b} / b$, or $\delta_{b} /(d-b)$ when the screen is close to the outer wall.

A similar condition has been given for a metallized ceramic wall [20], where $(d-b)$ is replaced by the thickness of the ceramic wall. In the transverse direction, the shielding condition under the same assumption becomes simply [21] $t / \delta_{b} \gg \beta^{2} \gamma^{2} \delta_{b} / b$, similar to the longitudinal criterion but without the logarithmic term. Hence for $\gamma$ not too large, shielding in the transverse direction is always achieved when longitudinal shielding is good.

For higher energies, taking into account the finite skin depth at the outer wall, the criterion becomes $t / \delta_{b} \gg(d / b)\left(\delta_{b} / \delta_{d}\right)$, i.e. the skin depth of the screen should be less than that of the outer wall. This is difficult to fulfill when the outer wall is ferro-magnetic and thus has a very small skin depth. However, at higher energies the beam is more stable and the space charge part of the impedance is strongly reduced.

\subsection{Longitudinal stability criteria}

The simplified Keil-Schnell or circle criterion is often used to estimate longitudinal stability limits, but is really not applicable for space charge dominated beams, since the actual stability limit is very large for a capacitive reactance. For bunched beams, one has to replace average current $I_{b}$ by peak current by dividing it with the bunching factor $B_{f}$. One thus obtains the Boussard criterion for the microwave instability

$$
\frac{\left|Z_{\|}\right|}{n}<F \frac{|\eta| B_{f} E_{0}}{e \beta^{2} I_{b}}\left[\frac{\Delta E}{E}\right]_{\text {FWHM }}^{2} .
$$

The form factor $F$ - originally assumed to be of the order of unity - is much larger for a space charge dominated impedance. In Phase I, the total beam current with $3.10^{13}$ protons is $1.4 \mathrm{~A}$ in 126 bunches and $I_{b}$ only $12 \mathrm{~mA}$. The bunching factor is about 0.5 near injection energy $E=\gamma E_{0}=1.3 \mathrm{GeV}, E_{0}$ being the rest energy. The full energy spread is then $7.10^{-4}$, and the full spread at half height about $\sqrt{2}$ smaller. With a transition gamma of $\gamma_{t}=27.71 j$ and $F=1$, the stability limit starts from $24.95 \mathrm{k} \Omega$ at injection and drops monotonically to 


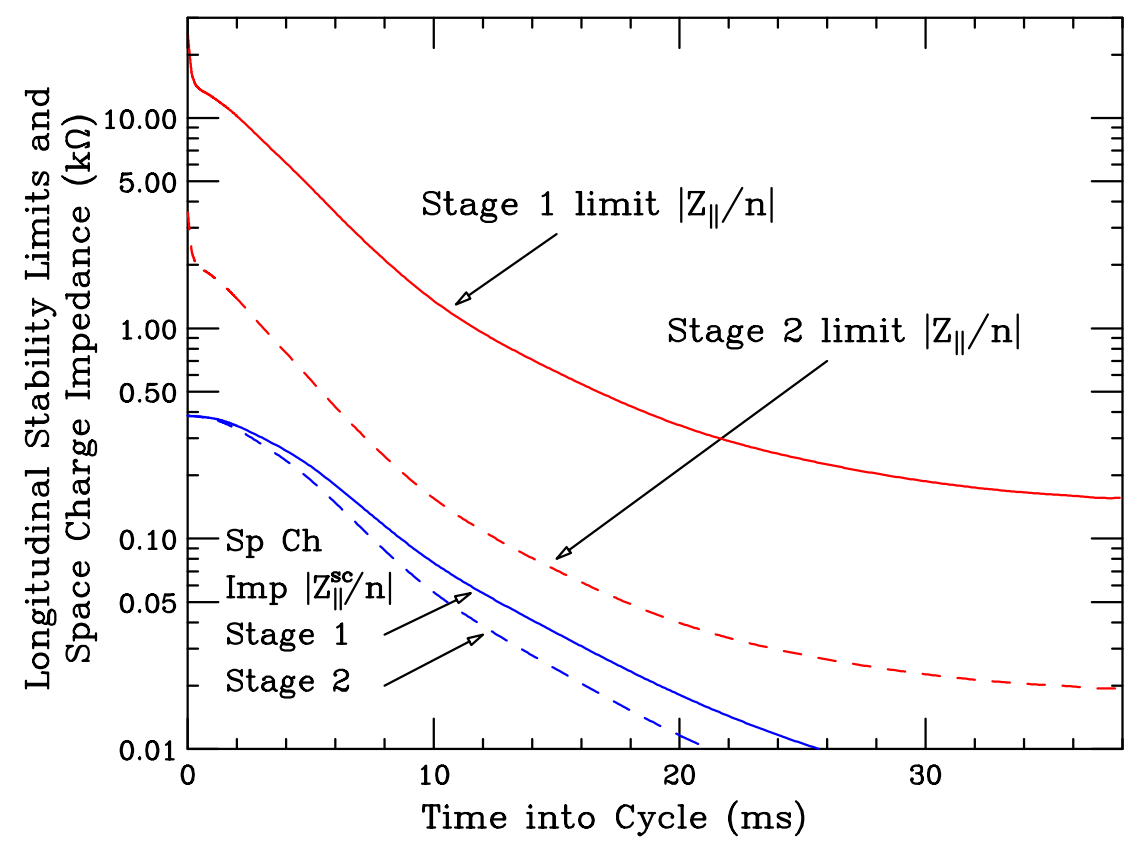

Figure 3: (color) Plot showing the longitudinal microwave stability limits of Phase I Stage 1 (solid red curve) and Stage 2 (dashed red curve) with form factor $F=1$ during the acceleration cycle. The corresponding space charge impedances per harmonic are shown as solid blue and dashed blue curves.

$0.16 \mathrm{k} \Omega$ at extraction (solid red curve in Fig. 3, and is at all times larger than the space charge impedance $\left|Z_{\|}^{s c} / n\right|$ (solid blue curve). For Phase I Stage 2, the same current is divided into only 18 bunches so that the threshold is 7 times smaller (red dashed curve), but is still much larger than $\left|Z_{\|}^{s c} / n\right|$ (blue dashed curve) at all times, and hence no problem is expected from microwave instability.

\subsection{Transverse stability criteria}

The Boussard-Keil-Schnell-like criterion for transverse bunched beam stability is (for $z=x, y)[18]$,

$$
\left|Z_{\perp_{z}}\right|<F \frac{4 B_{f} E}{e \beta\left\langle\beta_{z}\right\rangle I_{b}}\left[\frac{\Delta E}{E}\right]_{\mathrm{FWHM}}\left|S_{z}\right|,
$$

where the effective chromaticity is $S_{z}=\left(n-\nu_{z}\right) \eta+\xi_{z}$, with $n$ an arbitrary integer and $\xi_{z}$ the chromaticity. Instability occurs only for slow waves with $n>\nu_{z}$. The form factor $F$ depends on the transverse particle distribution, but is large compared to unity for a space charge dominated impedance as in the longitudinal case. Here, $\eta$ is always negative. If also the chromaticity $\xi_{z}$ is negative, the two terms add and cannot cancel. The lowest value of 
the spread is obtained for $n$ just above the tune $\nu_{z}$. Since also $|\eta| \approx \frac{1}{2}$, the first term in $S_{z}$ can be neglected if $\left|\xi_{z}\right|$ is large enough. For $F=\left|S_{z}\right|=1$, the transverse impedance threshold dips down to $11 \mathrm{M} \Omega / \mathrm{m}$ in Stage 1 about $5 \mathrm{~ms}$ into the acceleration cycle (solid red curve of upper or lower plot of Fig. 4). This is very much larger than $\left|Z_{\perp z}^{r w}\right|$ even when $|n-\nu|=1$, and requires $\xi_{z} \approx-4$ to cope with the space charge impedance (solid blue curve). In Stage 2, however, the threshold is 7 times smaller (red dashed curve) and is still much larger than $\left|Z_{\perp z}^{r w}\right|$, but requires $F\left|S_{z}\right| \approx 28$ to overcome the space charge impedance (dashed blue curve). To safeguard stability, one may blow up the longitudinal emittance so as to attain a larger energy spread, thus enhancing Landau damping.

\subsection{Cures}

Inductive inserts can be effective for compensating the "capacitive" space charge impedance. However, they require considerable space around the machine. Ferrite loaded cavities and inductive inserts may contribute a large broad band impedance, with a resistive part which becomes large at frequencies where the ferrites become lossy. This impedance can drive a longitudinal microwave instability such as the one observed at the LANL PSR [19]. Therefore, ferrite with small losses should be chosen to limit the resistive impedance. Another way to lower the resistive impedance and avoid microwave instability is to heat the ferrite insert to $100^{\circ} \mathrm{C}$ or $150^{\circ} \mathrm{C}[19]$.

It is further prudent to keep transition well above the highest operation energy by designing a lattice with small or imaginary momentum compaction. A reduction of transition to limit the required bunch rotation could be dangerous and should be applied only very shortly before the beam is ejected.

\section{COUPLED BUNCH INSTABILITIES}

\subsection{Narrow-band impedance estimates}

We already mentioned the resistive wall impedance, which is in particular high in the transverse plane at the lowest betatron frequency $q f_{0}$, which is about $100 \mathrm{KHz}$ for $q=0.33$. For a thick Inconel wall at $b=63 \mathrm{~mm}$, with a skin depth of $1 \mathrm{~mm}, Z_{\perp} \approx 200 \mathrm{k} \Omega / \mathrm{m}$, but for a very thin one, such as proposed for shielding the pole pieces, the value would be much higher, e.g 40 times for a thickness of $1 \mathrm{mil}=25 \mu \mathrm{m}$. For copper, with a nearly hundred 

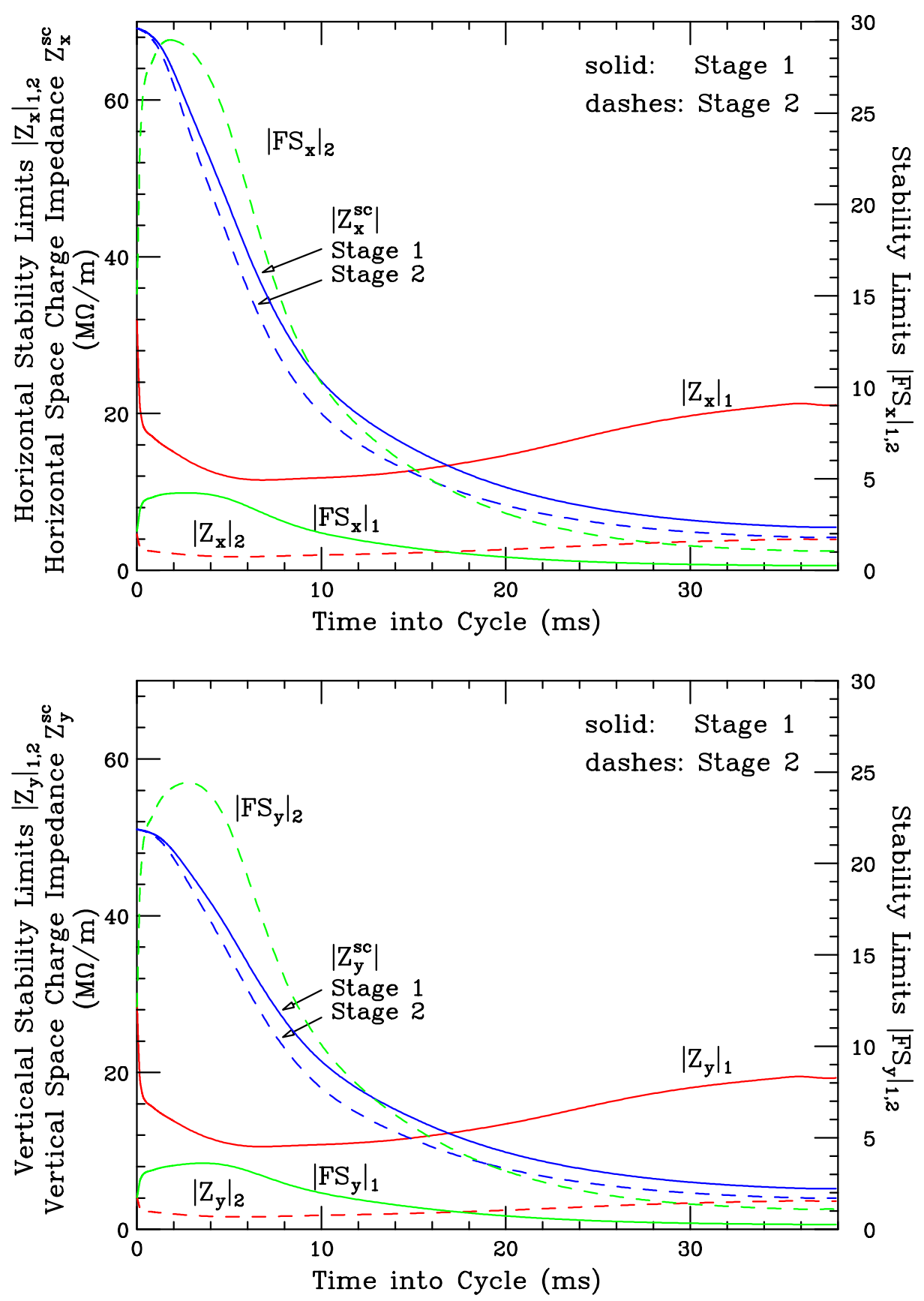

Figure 4: (color) Plots showing the transverse microwave stability limits of Phase I Stage 1 (solid red curve) and Stage 2 (dashed red curve) with form factor $F=1$ and effective chromaticity $S_{z}=1$ during the acceleration cycle. The corresponding space charge impedances are shown as solid blue and dashed blue curves. The amount of $\left|F S_{z}\right|$ required to cope with the space charge impedance to maintain stability are also shown (in green). Upper plot is for the horizontal while lower plot is for the vertical. 
times better conductivity, all values are 10 times lower.

In addition, we have to include narrow band resonances of higher order modes (HOMs) in rf and other cavities, such as kicker tanks for injection or ejection. For Stage 2 of Phase I, the $5 \mathrm{MHz}$ rf cavities will be either tuned or damped by ferrite (or Finemet) and their losses will damp most HOMs. However, for Stage 1, with a $53 \mathrm{MHz}$ rf system, the cavity modes must be measured and either damped internally or coupled out to a load. Measurements should be made when a prototype of these cavities becomes available.

Also the kicker tanks should be designed to permit damping of the HOMs by similar means. Sometimes it is already sufficient to use lossy material for the insulators inside these tanks.

\subsection{Longitudinal stability criterion}

Coupled-bunch modes will become unstable in a beam of $n_{b}$ equally space bunches with equal average currents $I_{b}$ when the imaginary part of the complex frequency shift $\mathcal{I m}_{m} \Delta \omega_{m, k}$ exceeds the frequency spread [17]. Here $m \geq 1$ is the azimuthal mode number $(m=1$ dipole, $m=2$ quadrupole mode etc.), and $0 \leq k<n_{b}$ the modal mode number of an oscillation with phase shift $\Delta \phi=2 \pi k / n_{b}$ between adjacent bunches [17]:

$$
\Delta \omega_{m}=\frac{2 m}{m+1} \frac{E_{0} I_{b}}{e h V_{\mathrm{rf}} \cos \phi_{s}}\left(\frac{Z_{\|}}{n}\right)_{\mathrm{eff}}^{(m, k)},
$$

where the effective impedance is defined as the (infinite) sum over the product of the impedance $Z_{\|} / n$ and the power density $g(\omega)$, evaluated at all spectral frequencies $\omega_{m k p}=$ $\omega_{0}\left(p n_{b}+k+m \nu_{s}\right)$, and normalized by the sum over all power densities:

$$
\left(\frac{Z_{\|}}{n}\right)_{\mathrm{eff}}^{(m, k)}=\sum_{p=-\infty}^{\infty} \frac{Z_{\|}}{n}\left(\omega_{m k p}\right) g\left(\omega_{m k p}\right)
$$

where the power spectrum $g(\omega)$ of the m-th mode of oscillation has been normalized such that $\sum_{p} g\left(\omega_{m k p}\right)=1$.

For a single resonance at frequency $\omega_{r}$, with shunt impedance $R_{s}$ and quality factor $Q$, the growth rate of the longitudinal coupled bunch oscillations can be written as

$$
\frac{1}{\tau}=-\frac{|\eta| n_{b} I_{b} R_{s} f_{0}}{2 \pi \nu_{s} B_{f}} \operatorname{Re}\left[D\left(\alpha_{d}\right) F_{m}(\Delta \Phi)\right]
$$


where $\alpha_{d}=\omega_{r} \tau_{\text {sep }} /(2 Q)$ is the decrement between bunches separated by $\tau_{\text {sep }}$. The function $D\left(\alpha_{d}\right)$ is unity for small arguments, and decreases rapidly for larger ones. The form factor $F_{m}$ is a function of the phase shift across the bunch $\Delta \Phi=\omega_{r} \tau_{L}$. It has maxima for the $m$-th mode when the argument is $m \pi$, decreasing approximately as $1 / m$.

The required damping of the higher modes in the rf cavities, kicker tanks and other incidental cavities can be calculated only when their frequencies and shunt impedances, shunt impedances and quality factors have been measured. Computer programs like BBI [23] or ZAP [24] can be used to perform the necessary summations over all impedances.

\subsection{Transverse stability criterion}

The transverse parasitic resonances in the $53 \mathrm{MHz}$ rf cavities can also drive transverse coupled-bunch instabilities in the same way as the longitudinal parasitic resonances. For $n_{b}$ bunches each with average current $I_{b}$, the growth rate is [18]

$$
\frac{1}{\tau}=-\frac{1}{m+1} \frac{e n_{b} I_{b} c}{4 \pi \nu_{z} E} \sum_{k} \operatorname{Re} Z_{\perp z}\left(\omega_{k}\right) F_{m}^{\prime}\left(\omega_{k}-\chi_{z}\right)
$$

where $\chi_{z}=-\xi_{z} \omega_{0} \tau_{L} / \eta$ is the chromatic phase shift across the full length $\tau_{L}$ of the bunch. The frequency spectrum for the $m$-th mode of transverse oscillations with coupled bunch mode number $n\left(0 \leq n<n_{b}\right)$ is given by $\omega_{k}=k n_{b}+n+\nu_{z}+m \nu_{s}$. The form factor $F^{\prime}(\omega)$ expresses the cancellation occurring in the summation over both positive and negative frequencies of the real part of the impedance, weighted by the spectrum of the $m$-th mode of oscillation.

Unlike the longitudinal situation, transverse coupled-bunch instabilities can also be driven by the resistive wall impedance at the sub-harmonic frequency $\left(\left[\nu_{z}\right]-1\right) f_{0}$, where $f_{0}$ is the revolution frequency and $\left[\nu_{z}\right]$ the noninteger part of the betatron tune. In this situation, however, the contribution is dominated by only one spectral line at the above low frequency and $F^{\prime} \approx 0.8$. This is because the next driving spectral line will be $n_{b} f_{0}$ away and the contribution will be much smaller because of the higher frequency.

Fortunately the Fermilab Proton Driver is small so that the driving resistive wall impedance, which scales as the ring circumference, is small also. With the resistive wall impedance computed in Sec. 3.1, the growth times are just $137 / 37 \mathrm{~ms}$ horizontally/vertically with $\xi_{z}=0$ at injection in both Stages 1 and 2. The growth rate for mode $m=0$ can be lowered by operating at a negative chromaticity. However, this may introduce instabilities of mode $m \geq 1$ 
which can be damped by a tune spread of order $1 /\left(\omega_{0} \tau_{L}\right)$ that usually occurs naturally; thus a low-frequency damper may not be necessary.

\subsection{Cures}

The design of the vacuum chamber and the choice of material for the chamber walls are most important to keep the resistive wall effect small. Sufficient shielding of the magnetic pole pieces is necessary if they form part of the vacuum chamber or are separated only by ceramic or composite walls.

The obvious cure for HOMs of the cavities is damping by lossy material inside or by coupling the offensive modes out into a load. A larger energy spread would increase the safety margin for Landau damping and could be obtained simply by increasing the (negative) chromaticity. The transverse feedback system would only be required for the lowest unstable oscillation frequencies and could thus be rather inexpensive.

\section{ELECTRON-PROTON INSTABILITY}

\subsection{Equations of motion}

When a proton beam is partially neutralized, with fractional neutralization $\chi_{e}$, the electrons in it will start to oscillate transversely with the so-called bounce frequency. For small amplitudes it is given by

$$
\omega_{e}^{2}=4 c^{2} r_{e} n_{p}
$$

where $r_{e}=1.53510^{-15} \mathrm{~m}$ is the classical electron radius, and $n_{p}=N_{p} /\left(\pi a_{x} a_{y} L_{b}\right)$ is the volume density of the protons in a bunch with $N_{p}$ protons, cross section $\pi a_{x} a_{y}$ and full length $L_{b}$.

The oscillating electrons will also excite the protons to oscillate with frequency

$$
\omega_{p}^{2}=4 c^{2} r_{p} n_{e}=4 c^{2} r_{p} \chi_{e} n_{p}
$$

The coupled oscillations lead to a dispersion relation for the e-p oscillation frequency $\omega$ as function of the azimuthal mode number $n$ :

$$
\left(\omega_{e}^{2}-\omega^{2}\right)\left[\omega_{\beta}^{2}+\omega_{p}^{2}\left(n \omega_{0}-\omega\right)^{2}\right]=\omega_{e}^{2} \omega_{p}^{2}
$$


The amplitudes of the lighter electrons will grow rapidly, while the oscillations of the heavier proton remain smaller. When electrons reach the vacuum chamber wall, more electrons may be generated by secondary emission which can lead to an avalanche effect called multipactor. This may then lead also to emittance growth or beam loss of the protons.

\subsection{Observations}

e-p oscillations have been seen in a number of proton storage rings (PSRs), and were overcome by different means. In a small PSR at the INP in Novosibirsk, constructed mainly for the study of charge exchange injection, it could be overcome by a simple feedback system. In the CERN ISR, electrons impinging on the chamber wall caused periodic background spikes, and had to be eliminated by better clearing and pumping. However, at the PSR in Los Alamos (LANL), the e-p instability was limiting the beam current for many years to values below the design goal. All attempts to eliminate or at least reduce the number of electrons failed.

The most common method to reduce neutralization of a beam is to leave a gap in the train of bunches. A more active method is to install clearing electrodes, and eliminate the electrons by applying transverse electric fields. If the electrons are generated by vacuum, better pumping may help-but not if the electrons are coming from the $H^{-}$stripping foil. Multipactor at the wall can be reduced by coating with a material with low secondary emission coefficient, such as Ti-N. If nothing else helps, a feedback system can be the solution. For the case of the LANL PSR, all these methods were tried and failed, and only a combination of higher rf voltage, sextupoles, partial wall coating and finally inductive insert permitted to reach the design current.

A particular encouraging experience comes from the spallation source ISIS, where no e-p instability has ever been seen, even when the vacuum pressure was increased by switching off several pumps. The instability did not even occur when the machine was not running as a rapid-cycling synchrotron but with stored beam. This observation is not fully understood, and further studies are being made, both theoretically and experimentally.

For the present Proton Driver, the injection from the linac is in chopped beams. As the rf voltage is raised, the bunching factor decreases rapidly from $B_{f}=0.9$ to 0.44 in $2 \mathrm{~ms}$ and 0.18 one half into the acceleration cycle. Thus, there will be large bunch gaps so that trapped electrons should be cleared and hence no e-p instability is expected. 
There has been some argument that a very low vacuum is mainly required for storage rings, not for a rapid cycling synchrotron as the Proton Driver where the beam is present only for a short time. This argument, unfortunately, is not quite correct. Take the LANL PSR, for example. The injection from the linac is generally accumulated for 1000 to 2000 PSR turns before the whole beam is extracted. The revolution period of the PSR is $0.358 \mu \mathrm{s}$. This gives a total accumulated or storage time of 0.36 to $0.72 \mathrm{~ms}$. The longest accumulated time ever accomplished in the PSR has been $1.225 \mathrm{~ms}$, the maximum obtainable at $1 \mathrm{~Hz}$ from the linac. In any case, the accumulated or storage time at the PSR is very much less than the acceleration cycle of $38 \mathrm{~ms}$ for the Proton Driver. However, this does not necessarily imply the requirement of a low vacuum in the Proton Driver, since electrons are created by many other processes in addition to rest gas ionization. While the vacuum inside the vacuum chamber of the LANL PSR is 2 to $4 \times 10^{-8}$ Torr, a vacuum at level of $10^{-7}$ Torr is generally considered economically achievable for the Proton Driver, even with the magnets inside the vacuum, which has a rather high out-gassing rate due to various exposed epoxy surfaces. This approach - with shielding by a liner made from copper strips to reduce the impedance of the exposed ferro-magnetic pole pieces while keeping eddy current losses small, is presently considered the preferred solution for the vacuum chamber of the Fermilab Proton Driver.

\section{References}

[1] J. Laslett, BNL Report 7534, (1963) p. 234.

[2] Z. Qian, private communication.

[3] C. Prior, private communication..

[4] R. Baartman, AIP Conf. Proc. 448 (1998) p. 56.

[5] A. Chao, M. Tigner, Handbook Accel. physics, World Scientific (1999).

[6] A. Chao, Physics of Collective Beam Instabilities, Wiley (1993).

[7] J. Norem et al, Proc. PAC Vancouver 1995, p. 396; D. Trbojevic etal, Proc. PAC Vancouver, 1995, p. 1030.

[8] J. MacLachlan et al, Fermilab Report 1650, 1990.

[9] M.A. Plum, et. al., Phys. Rev. ST Accel. Beams, 2, 064201 (1999). K.Y. Ng, J.E. Griffin, D. Wildman, M. Popovic, A. Browman, D. Fitzgerald, R. Macek, M. Plum, and T. Spickermann, Proceedings of the Particle Accelerator Conference, June 18-22, Chicago, 2001.

[10] K.Y. Ng, Fermilab Report FN-702, 2000. 
[11] K.M. Fung, M. Ball, C.M. Chu, B. Hamilton, S.Y. Lee, and K.Y. Ng, Phys. Rev. ST Accel. Beams 3, 100101 (2000).

[12] I.M. Kapchinskij and V.V. Vladimirskij, Proc. 2nd Int. Conf. on High Energy Accel. and Instr., CERN, Geneva, 1959, p. 274.

[13] K.Y. Ng, Space-Charge Effects on Bunch Rotation, Fermilab Report FN-702, 2001. 5133 (1998).

[14] C. Prior, Track-2D, private communication.

[15] F. Caspers, Proc. PAC New York, 1999, p. 1408.

[16] I. Gardner et al, Part. Accel. 31, 227 (1990).

[17] F. Sacherer, CERN Report CERN/MPS/BR 73-1, 1973; IEEE-NS 20, 3, 825 (1973).

[18] F. Sacherer, CERN Report 77-13, 1977.

[19] K.Y. Ng, J.E. Griffin, D. Wildman, M. Popovic, A. Browman, D. Fitzgerald, R. Macek, M. Plum, and T. Spickermann, Proceedings of the Particle Accelerator Conference, June 18-22, Chicago, 2001.

[20] A. Piwinski, DESY Report 1978.

[21] R. Gluckstern, US Accelerator School, Phoenix, AZ, Jan. 2000, to be published as CERN Yellow Report.

[22] D. Moehl, A. Sessler, LBL Report 1970.

[23] A. Hofmann et al, IEEE-NS 26, 3514 (1979).

[24] M. Zisman et al, LBL Report 21270, 1985. 